\title{
FUERZA MUSCULAR Y FLEXIBILIDAD DE JUDOKAS MASCULINOS DE ALTO RENDIMIENTO QUE PARTICIPARON EN LA LIGA ESPAÑOLA DURANTE 2011
}

\section{MUSCLE STRENGTH AND FLEXIBILITY OF MALE JUDO ATHLETES IN HIGH-PERFORMANCE PARTICIPANTS OF THE SPANISH CHAMPIONSHIP IN 2011}

\author{
Alves Kimo, João ${ }^{1}$; Borba-Pinheiro, Cláudio Joaquim ${ }^{2,3,4}$; Gurgel de Alencar Carvalho, Mauro César 2,5; Chulvi-Medrano, Iván ${ }^{1}$; \\ Dantas Martin, Estélio Henrique ${ }^{2}$.
}

1. Universidad de Valencia, Valencia, Espanha

2. Programa de Pós Graduação Stricto Sensu em Enfermagem e Biociências (PPGEnfBio/Doutorado) da Universidade Federal do Estado do Rio de Janeiro (UNIRIO). Rio de Janeiro, Brasil.

3 Universidade do Estado do Pará (UEPA). Laboratório de Treinamento Resistido com Ênfase em Saúde (LERES). Campus XIII, Pará, Brasil.

4 Instituto Federal do Pará (IFPA) Campus de Tucuruí, Pará, Brasil.

5 Colégio Pedro II (Autarquia Federal) Rio de Janeiro, Brasil.

ALVES, K.J.; BORBA-PINHEIRO, C.J.; GURGEL DE ALENCAR, C.M.C.; CHULVI-MEDRANO, I.; DANTAS M.E.H. Fuerza muscular y flexibilidad de judokas masculinos de alto rendimiento que participaron en la liga española durante 2011. Mot. Hum. 13(1): 28-35; 2012.

\begin{abstract}
RESUMEN
El objetivo fue evaluar los efectos de un programa de entrenamiento de judo sobre la fuerza muscular de los brazos y región lumbar junto a la flexibilidad lumbo-pélvica y la posible correlación entre estas variables sobre el rendimiento deportivo del judo en judokas españoles. El estudio incluyó 14 atletas varones de alto rendimiento de judo con $19.5 \pm 2.9$ años y 66.1 $\pm 11.8 \mathrm{~kg}$ de masa corporal. Los instrumentos para la evaluación fueron: dinamómetro de fuerza para los músculos de los miembros superiores y región lumbar y el cajón diseñados específicamente para el test de flexibilidad conocido como sitand-reach. El periodo de entrenamiento comprendió siete semanas de preparación física y entrenamiento técnico, con variación de intensidades en periodos alternativos. A los resultados se les aplicó tratamientos estadísticos paramétricos y no paramétricos. El análisis de los resultados mostró un incremento significativo sobre los músculos de la región lumbar ( $\Delta \%$ $=40,8 \%, p=0,0001)$, músculos de los brazos $(\Delta \%=48,2 \%, p=0,0006)$ y flexibilidad lumbar $(\Delta \%=25,2 \%, p<0,0001)$. Adicionalmente, no fue hallada correlación entre las variables antropométricas y la edad en relación con el porcentaje de ganancia de fuerza y flexibilidad. Sin embargo, los aspectos que determinan las ganancias de fuerza de los músculos del brazo y de la región lumbar parecen ser los mismos, donde $r=0,71, p=0,023$, mientras que los aspectos que determinan los incrementos de la flexibilidad no parecen ser los mismos que favorecen las ganancias de fuerza $(-0,118>r>-0,302$ y $0,74>p>0,37)$. Los resultados del presente estudio muestran un incremento en las ganancias de fuerza y flexibilidad, encontrándose adicionalmente una correlación entre las variables de fuerza para el desarrollo y desempeño atlético-motor de alto rendimiento de judo.
\end{abstract}

Palabras clave: Fuerza, Movimiento articular, Rendimiento atlético, Entrenamiento de judo.

\begin{abstract}
The objective To evaluate the effects of a judo training program on muscle strength of arms, lumbar muscles, flexibility and the possible correlation between these variables in judo high performance of Spain. The study considered 14 male athletes of high-performance with $19.5 \pm 2.9$ years and $66.1 \pm 11.8 \mathrm{~kg}$ of body mass. The instruments for evaluation were: dynamometer for force of arms and lumbar muscles, and flexibility test of the sit and reach. The plan had a period of seven weeks of technical and physical training, with varying intensities in alternate periods. For data analysis parametric and nonparametric tests were used. The study variables showed a significant increase: lumbar $(\Delta \%=40.8 \%, p=$ $0.0001)$, arms $(\Delta \%=48.2 \%, p=0.0006)$ and flexibility $(\Delta \%=25.2 \%, p<0.0001)$. In addition, no significant correlation between anthropometric variables and age when related to the percentage gains in strength and flexibility. However, the aspects that determine the strength of arm and muscles lumbar gains seem to be the same, where $r=0.71, p=0.023$, while the aspects that determine the flexibility gains does not seem to be the same forces that promote, where -0.118> $r>$ -0.302 and $0.74>p>0.37$. The study indicates an improvement in the variables of strength and flexibility, was also found a correlation between the variables of force for the development of judo in high-performance.
\end{abstract}

Key words: Strength, Movement width to articulate, Athletic performance, judo training. 
ALVES, K.J.; BORBA-PINHEIRO, C.J.; GURGEL DE ALENCAR, C.M.C.; CHULVI-MEDRANO, I.; DANTAS M.E.H. Fuerza muscular y flexibilidad de judokas masculinos de alto rendimiento que participaron en la liga española durante 2011. Mot. Hum. 13(1): 28-35; 2012.

\section{INTRODUCCIÓN}

El judo de alto rendimiento es una modalidad que requiere el desarrollo de diversas capacidades y habilidades físicas con demandas elevadas, entre ellas, la fuerza muscular y la flexibilidad, debido a la necesidad de rendimiento motor y de protección ante lesiones durante las competiciones (1). Para Verkhohanscki (2) los deportes de combate están caracterizados por la compleja diversidad de acciones motoras en diferentes condiciones de estrés con una gran variabilidades de la intensidad y de fatiga. De este modo, el entrenamiento de judo de alto rendimiento requiere un planteamiento específico con atención a las variables que influyen en el resultado de rendimiento de los atletas (3).

La necesidad de información fidedigna relacionada con el acondicionamiento físico de atletas de élite ha llevado a la comunidad científica a estudiar las diversas variables de rendimiento específico de los atletas de diferentes modalidades de lucha (4). Consecuentemente, se pueden observar una preocupación común en los estudios vinculados al judo, donde se pone de manifiesto que el éxito o fracaso de la competición está muy determinado con los condicionantes vinculados a la fuerza muscular $(5,6,7,8)$.

La investigación relacionada con el rendimiento de atletas de judo son relativamente recientes dentro de la comunidad científica y evidencian los factores influyentes en el rendimiento físico final de los competidores de élite, pudiendo destacar: a) la necesidad de una evaluación/ diagnóstico del estado inicial del judoka; b) la planificación y periodización del entrenamiento bien sea para evitar lesiones como para incrementar el rendimiento de la fuerza muscular, la flexibilidad, el acondicionamiento aeróbico y anaeróbico $(6,7,8,9)$. Atendiendo a lo anteriormente expuesto, las cuestiones que el estudio tiene como objetivo contestar son: 1) ¿Puede un programa de fuerza específico para atletas de judo de élite mejorar la fuerza de sus brazos, fortalecer los músculos de la espalda e incrementar la flexibilidad de la región lumbar? 2) ¿Existe una correlación entra estas tres variables?

Consecuentemente, el objetivo general del estudio es registrar los efectos de un programa de entrenamiento de fuerza específico sobre la fuerza de brazos, la fuerza lumbar, la flexibilidad y su posible correlación entre judocas de élite de España.

\section{MATERIAL Y MÉTODOS}

\section{Muestra}

La muestra estuvo conformada por 14 judokas varones con $19,5 \pm 2,9$ años de edad; $66,1 \pm 11,8$ de masa corporal y $168,5 \pm 11,5$ de talla. Además, los atletas eran de diferentes categorías de peso $(-60 \mathrm{~kg}$ a $-90 \mathrm{~kg})$. Todos los deportistas participaron en la liga española durante el primer semestre de 2011. Todos los sujetos fueron reclutados del centro de alto rendimiento conocido como Centro de Entrenamiento (CT) de Valencia Terra y Mar (Valencia, España). A la hora de seleccionar la muestra se mantuvieron los siguientes criterios de inclusión: a) ser varón; b) haber ganado torneos clasificatorios para el campeonato nacional en España. Todos los sujetos se prestaron voluntarios para la investigación firmando un consentimiento informado, tras la explicación de los procedimientos experimentales y aclaración de dudas particulares, siguiendo las recomendaciones para la realización de investigaciones en seres humanos (10).

\section{Procedimientos de Intervención. Evaluación de la composición corporal.}

Inicialmente se realizó una evaluación de la composición corporal de la muestra utilizando para ello una báscula Filizola ${ }^{\circledR}$ escala antropométrica (Brasil) con una capacidad de 150 kilogramos y rangos de 100 gramos. Con el sujeto descalzo y con ropa ligera, se colocaba encima de la báscula con los talones juntos y la cabeza colocada en el plano horizontal. Esta misma posición fue utilizada para evaluar la estatura, para lo que utilizó el estadiómetro vertical fijado en la báscula. Con estas variables registradas, se calculó el índice de masa corporal (IMC). Para determinar el porcentaje de grasa corporal, se utilizó una bioimpendancia (OMROM BF306® [España]).

Para asegurar la validez de estas mediciones, el investigador principal ejecutó las valoraciones en conformidad con los requisitos de las normas internacionales para la evaluación antropométrica (11).

\section{Evaluación de la flexibilidad}

La flexibilidad de los judocas fue evaluada con la prueba de sit-and-reach, utilizando un cajón de Wells Sanny® (Brasil) (12). Este dispositivo que consiste en un cajón con medidas predeterminadas $30.5 \mathrm{~cm}$ x $30.5 \mathrm{~cm}$ x 30.5 $\mathrm{cm}$, con un añadido de $23 \mathrm{~cm}$ para el apoyo de los miembros superiores. En la cara superior del cajón aparece una escala métrica de $50 \mathrm{~cm}$ que permitirá cuantificar el nivel de flexibilidad, en función del alcance del sujeto desde la posición de partida hasta la posición final lograda.

\section{Evaluación de la fuerza del brazo}

La evaluación de la fuerza del miembro superiores fue realizado mediantes un dinamómetro digital GRAM ${ }^{\circledR}$ CR-200 (España) aprobado por la Unión Europa. El dinamómetro se anclaba por un extremo a una barra fija y por otro lado a una barra que serviría de asidero para 
que el sujeto pudiera ejecutar una flexión de codo de 90 grados máxima isométrica desde la posición de bipedestación. Previo a cada valoración el dinamómetro era calibrado para asegurar su validez. La fuerza máxima isométrica se obtenía manteniendo 7 segundos la contracción en la posición descrita anteriormente, fueron realizados 3 intentos, permitiendo un descanso suficiente entre pruebas, y se registros la mejor marca de las tres realizadas. Este tipo de evaluación ha sido sugerido previamente por Franchini et al. (8) y Sánchez et al. (13) como una valoración útil para atletas de alto rendimiento en la disciplina del judo.

\section{Evaluación de los músculos lumbares}

Para evaluar la fuerza extensora de la región lumbar, fue anclado un extremo del dinamómetro digital GRAM $®$ CR-200 (España) a una barra fija y el otro extremo a un cinturón inextensible que era colocado alrededor del tronco del sujeto, por debajo del nivel de las axilas. El sujeto instrumentado se sentaba en una silla desde donde ejecutaría la extensión isométrica máxima. El protocolo de medición fue idéntico al llevado a cabo para los miembros superiores, así pues, mantuvieron 7 segundos el esfuerzo máximo isométrico, realizándose 3 intentos, separados en el tiempo lo suficiente como para garantizar la entera recuperación y de los 3 intentos fue seleccionada la mejora marca. Esta prueba ha sido recomendada para deportistas de artes marciales por O’Donovan et al. (14).

El estudio se llevó a cabo durante un periodo de siete semanas comprendiendo los meses de febrero y marzo del año 2011. Todos los sujetos mantuvieron su plan de entrenamiento específico del deporte consistente 120 minutos de entrenamiento 5 días a la semana.
Los sujetos involucrados en la investigación añadieron un programa de entrenamiento físico a razón de dos días semanales, los cuales fueron alternos.

Entrenamiento cardio-respiratorio 2 días a la semana de baja intensidad durante 25 minutos pudiendo realizarse en cinta de correr, remo estático o elíptica.

Entrenamiento de la fuerza. Los ejercicios fueran ejecutados al $80 \%$ de una repetición máxima (1RM) siguiendo el método alternado por seguimiento (15), 3 días a la semana, con una duración de 60 minutos. Paralelamente fueron realizados ejercicios funcionales ejecutados con fitballs y cuerdas para el entrenamiento de los extensores lumbares y flexores de brazo. Además fueron realizados ejercicios en la barra fija adaptada que también se utilizó para evaluar la fuerza de agarre en el judogui y entrenamiento de trepa en cuerda.

Todas las sesiones de entrenamiento fueron precedidas de un calentamiento general y estandarizada y finalizadas con una tanda de estiramientos, que fue repetida a la conclusión del entrenamiento. Los estiramientos fueron ejecutados estáticamente manteniendo la posición final durante 10 segundos para cada uno de los grandes grupos muscular. Para controlar la intensidad de los estiramientos se aplicó la escala basada en la percepción del esfuerzo de flexibilidad (PERFLEX) (16), con los parámetros de la escala se puede definir que los estiramientos fueron ejecutados entre los niveles 31 y 60 que corresponde a niveles de intensidad sub-máxima, como se muestra en la Figura 1.

\begin{tabular}{|lc|}
\hline Técnico & 5 días $/ \mathrm{semana}(120 \mathrm{~min} * 5=600 \mathrm{~min})$ \\
\hline Cardio-suave & 2 días $/ \mathrm{semana}(85 \min * 2=170 \mathrm{~min})$ \\
\hline $\mathbf{R T}+$ Físico Aeróbico + Entrenamiento funcional & 3 días $/ \mathrm{semana}(60 \mathrm{~min} * 3=180 \mathrm{~min})$ \\
\hline & $\mathbf{9 5 0}$ min de entrenamiento total a la semana \\
\hline
\end{tabular}

Tal y como recomienda el ACSM (2009) todas las evaluaciones y los entrenamientos fueron supervisados y monitorizados por profesionales cualificados, siendo en este caso el investigador principal el encargado de esta tarea.

\section{Análisis estadístico.}

Para el estudio estadístico se utilizó el programa Statistica ${ }^{\circledR}$ 7.0. Inicialmente se realizó un análisis descriptivo de las variables de la muestra estudiada extrayendo las medidas de tendencia central y de dispersión. La evaluación de la normalidad de los datos se llevó a cabo mediante el test de Shapiro - Wilk que demostró una distribución normal, por lo tanto, para el estudio de las diferencias entre variables se aplicó un estudio T - Student con medidas repetidas. Con el objetivo de determinar los porcentajes de ganancia de fuerza y de flexibilidad entre las 
ALVES, K.J.; BORBA-PINHEIRO, C.J.; GURGEL DE ALENCAR, C.M.C.; CHULVI-MEDRANO, I.; DANTAS M.E.H. Fuerza muscular y flexibilidad de judokas masculinos de alto rendimiento que participaron en la liga española durante 2011. Mot. Hum. 13(1): 28-35; 2012.

variables se aplicó el test de Kruskal Wallis, puesto que eran datos no paramétricos. Por último, las diferencias porcentuales fueron calculadas aplicando la formula $\Delta \%$ $=[100 *$ retest $) /$ prueba)-100] de las variables estudiadas. Para todos los análisis estadísticos se asumió un nivel alfa de $\mathrm{p}<0.05$ como poder significativo.

\section{RESULTADOS}

La tabla I presenta las características de la muestra de judokas estudiadas.

\begin{tabular}{|c|c|c|c|c|}
\hline \multicolumn{5}{|c|}{ Judokas Masculinos; n=14 } \\
\hline Variables & Media & $\mathrm{DE}$ & № Mín & № Máx \\
\hline Edad (años) & 19,5 & 2,9 & 16,0 & 25,0 \\
\hline Masa (kg) & 66,1 & 11,8 & 50,0 & 92,0 \\
\hline Talla (cm) & 168,5 & 11,5 & 150,0 & 190,0 \\
\hline$\%$ Grasa $(\%)$ & 19,3 & 6,1 & 10,5 & $29,45^{\circ}$ \\
\hline IMC (kg/m2) & 26,3 & 7,9 & 16,1 & 44,5 \\
\hline Flexibilidad (cm) & 24,5 & 6,9 & 12,2 & 34,4 \\
\hline Fuerza brazo (kgf) & 43,0 & 15,5 & 16,0 & 65,0 \\
\hline Fuerza Lumbar (kgf) & 94,4 & 47,1 & 31,0 & 180,3 \\
\hline \multicolumn{5}{|c|}{ DE= Desviación Estándar; $\mathrm{N}^{\circ}$ Mín= Número Mínimo ; $\mathrm{N}^{\circ} \mathrm{Máx}=$ Número Máximo; Rep=Repetición } \\
\hline
\end{tabular}

La figura 2 muestra un incremento significativo $(\mathrm{p}<0,05)$ en la fuerza muscular de la región lumbar $\Delta \%=40,8 \%$ cuando son comparados los valores del pre-test con los del post-test tras la intervención con el entrenamiento.

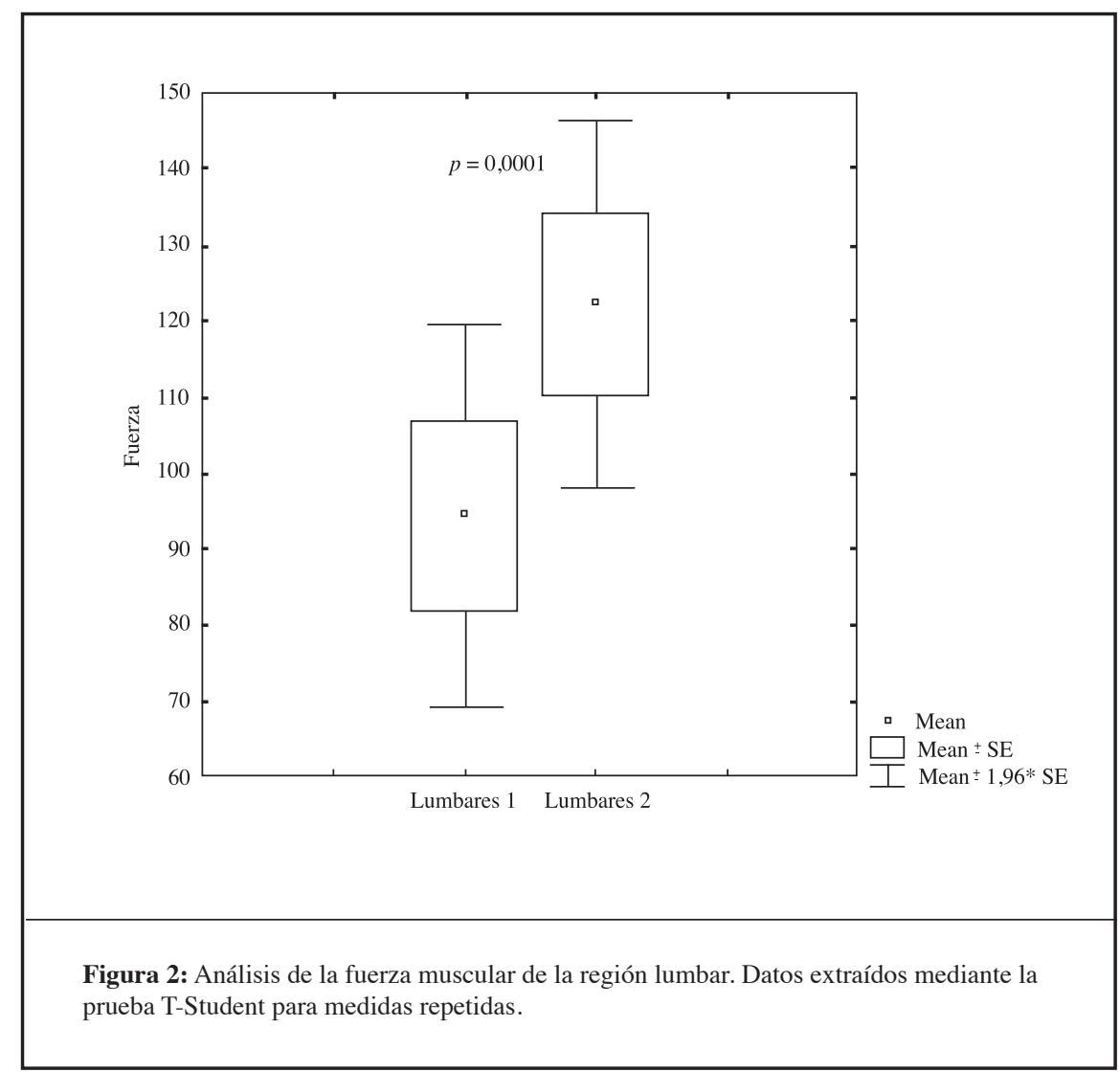


ALVES, K.J.; BORBA-PINHEIRO, C.J.; GURGEL DE ALENCAR, C.M.C.; CHULVI-MEDRANO, I.; DANTAS M.E.H. Fuerza muscular y flexibilidad de judokas masculinos de alto rendimiento que participaron en la liga española durante 2011. Mot. Hum. 13(1): 28-35; 2012.

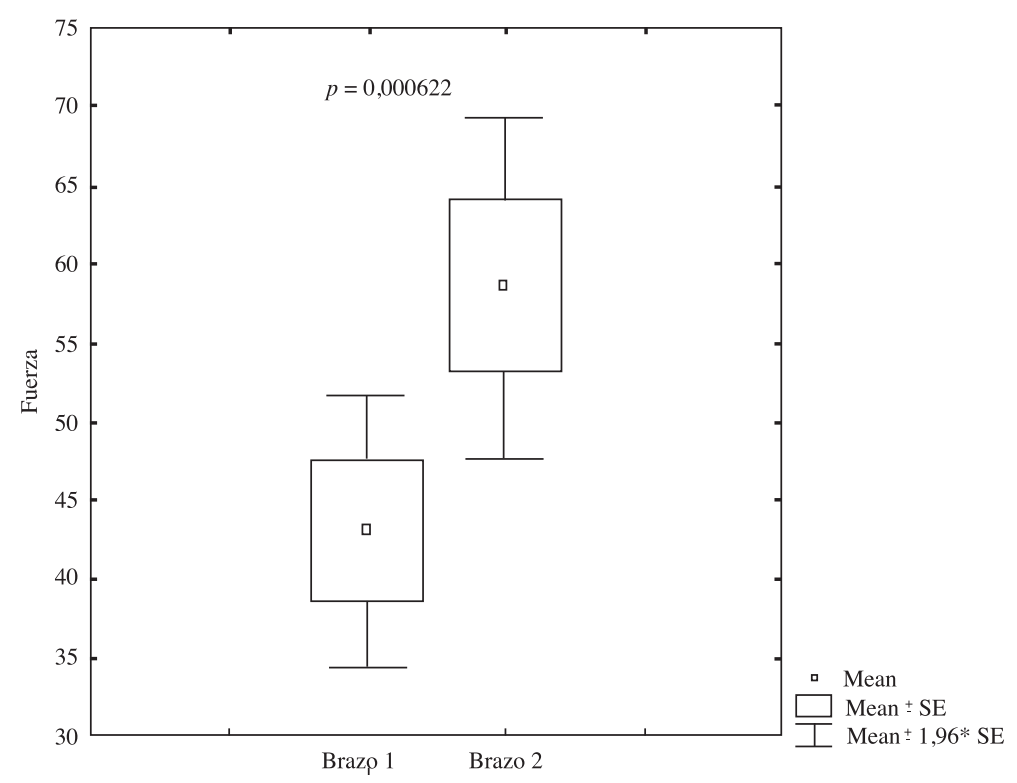

Figura 3: Análisis de la fuerza muscular de los brazos comparando los valores pre-test y post-test mediante un análisis T-Student para medidas repetidas.

La figura 3 muestra una incremento significativo de la fuerza de los brazos $[\Delta \%=48,2 \%(\mathrm{p}<0,05)]$. En la figura 4 se aprecian mejoras significativas en los valores de flexibilidad al finalizar el protocolo de entrenamiento $[\Delta \%=25,2 \%(\mathrm{p}<0,05)]$.

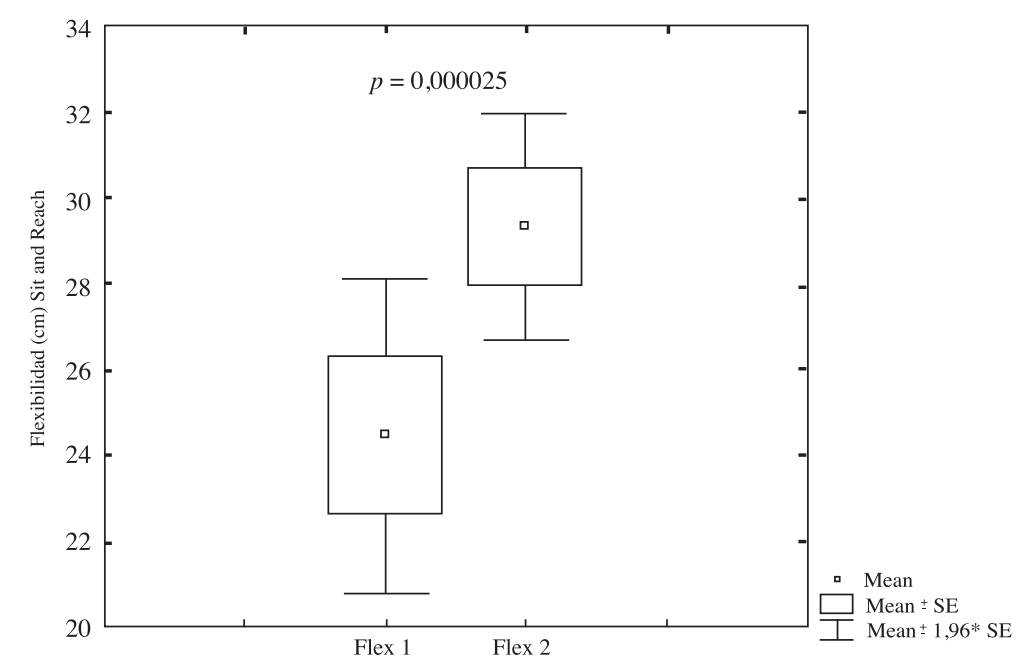

Figura 4: Valores de flexibilidad en la prueba de sit-and-reach. 
ALVES, K.J.; BORBA-PINHEIRO, C.J.; GURGEL DE ALENCAR, C.M.C.; CHULVI-MEDRANO, I.; DANTAS M.E.H. Fuerza muscular y flexibilidad de judokas masculinos de alto rendimiento que participaron en la liga española durante 2011. Mot. Hum. 13(1): 28-35; 2012.

La figura 5 representa una comparativa entre los porcentajes de ganancia de las variables analizadas (fuerza de brazos, fuerza en la región lumbar y flexibilidad). E puede apreciar que las mayores ganancias se produjeron en la fuerza de brazos y de la región lumbar. No obstante, debe ser destacado que no existieron diferencias significativas entre dichas ganancias donde $\mathrm{p}=1,96$.

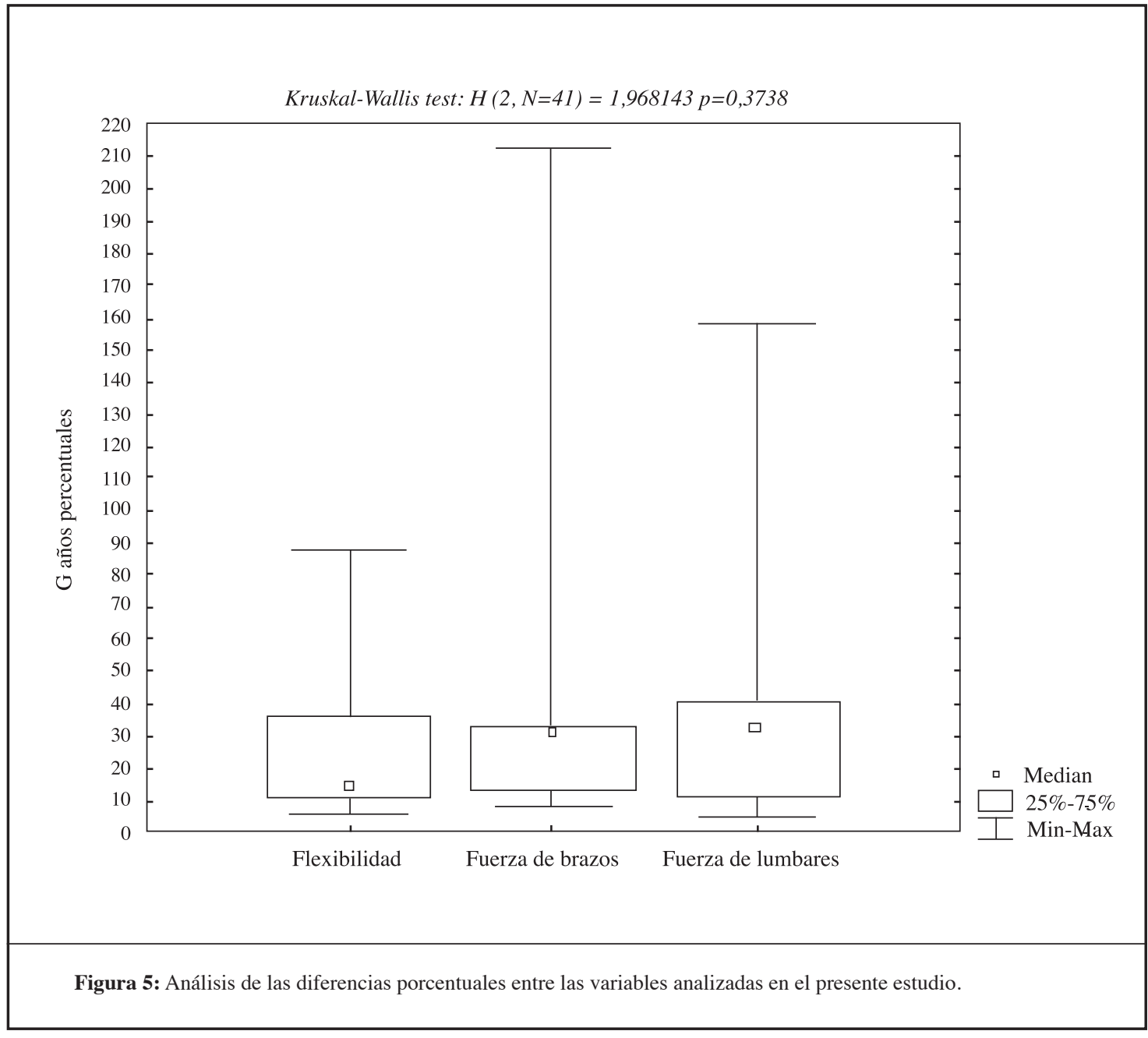

Los resultados mostraron que las variables antropométricas y la edad no ejercieron influencia alguna en las ganancias porcentuales de la fuerza y la flexibilidad, puesto que no apareció correlación significativa entre ellos.

Los aspectos que determinan las ganancias de fuerza en el brazo y en la musculatura lumbar parecen ser los mismos puesto que existió una fuerte correlación $\mathrm{r}=0,71 \mathrm{p}=0,023$, mientras que los aspectos que determinan las ganancias de flexibilidad no parece ser los mismos que aquellos la fuerza donde $-0,118>\mathrm{r}>-0,302$ y $0,74>\mathrm{p}>0,37$.

\section{DISCUSIÓN}

El presente estudio pretendió responder a dos objetivos. En primer lugar, conocer si el entrenamiento específico propuesto para judo de alto rendimiento puede incrementa la fuerza de brazos y de la región lumbar de forma conjunta al incremento de la flexibilidad. El segundo objetivo al que se pretendió responde fue conocer si existía relación entre las variables de fuerza de brazos, fuerza de la región lumbar y flexibilidad de la región lumbar. 
En respuesta a la primera cuestión, el estudio demostró que el entrenamiento resultó efectivo para el incremento de los rendimientos específicos de las tres variables (fuerza de brazos y de la región lumbar, incremento de la flexibilidad de la región lumbar), y aunque en principio parezca previsible, muestra una gran importancia para atletas de alto nivel que ya presentan un buen rendimiento de fuerza y flexibilidad.

La importancia de la fuerza en los judokas de alto rendimiento ha sido establecido previamente por Franchini et al. (8) quienes mostraron que los judokas de alto rendimiento obtenían mejores rendimientos en una prueba de evaluación de la resistencia a la fuerza dinámica, mediante un test de suspensión en barra con agarre en judogui (12 \pm 5 repeticiones) frente a los judokas de nivel regional ( $9 \pm 4$ repeticiones). Estos datos fueron corroborados en un trabajo posterior donde se puso de manifiesto que la fuerza de brazos era mayor en deportistas de alto rendimiento $(\Delta \%=48,2 \% ; \mathrm{p}=0,0006)$.

En el estudio llevado a cabo por Sánchez (13) fue evaluada la fuerza de prensión bilateral de 102 judokas con edades comprendidas de entre 15 y 19 años que participaban en la junior league de Galicia en España durante el año 2008. Sus pruebas determinaron la existencia de diferencias significativas entre las categorías masculinas de mayor peso. Adicionalmente detectaron la existencia de diferencias significativas en función de la altura del agarre, entre las mujeres judokas del estudio. A causa de falta de relación entre ambos parámetros, no se llega a considerar un factor de extrema importancia para la preparación específica.

El estudio de Sterkowicz et al. (17) desveló que la fuerza relativa de los músculos extensores de la rodilla en jóvenes judokas de Polonia era mayor que en los grupos sedentarios, no observando esta diferencia cuando se evalúa la fuerza flexo-extensora del tronco. Estos resultados llevaron a Sterkowicz a concluir que la fuerza relativa del judo de alto rendimiento era similar al de los grupos de no entrenados, incluso tras los muchos años de entrenamiento.

Los judokas muestran más fuerza en los músculos activos durante la competición, especialmente de los encargados en las acciones de proyección. Atendiendo a los músculos paravertebrales, especialmente en la región lumbar, es conocido son ampliamente utilizados en las acciones deportivas del judo, especialmente en las tareas defensivas para que no hayan fugas y mantener el equilibrio estático-dinámico, como en la parte ofensiva para la ejecución de proyecciones. Así pues, este estudió incluyo la valoración de la fuerza lumbar, donde se aprecia que el entrenamiento propuesto permitió un incremento significativo en los niveles de fuerza $(p<0,05)$. Consecuentemente, la mejora de las variables de fuerza específica y flexibilidad de la región lumbar puede implicar un factor decisivo para el rendimiento final de las competiciones y / o profilaxis de lesiones $(9,14)$.

No obstante, debe asumirse que la complejidad dinámica que entrañan los combates de judo (2) puesto que los deportes de lucha están caracterizados en particular por la variabilidad de las acciones motrices en el estrés de diversas intensidades $(2,18)$. Esta situación implica que el entrenamiento de fuerza no debería separarse del entrenamiento técnico. Bajo esta premisa Blais y Trilles (5) estudiaron el tiempo de ejecución de dos técnicas de proyección (O Soto Gari y Morote Seoi Nague) tras un periodo de entrenamiento de fuerza combinado con la técnica ,los resultados mostraron una mejoría significativa en el tiempo de ejecución de estas técnicas $(\mathrm{p}<0,05)$.

Por otro lado, la existencia de una correlación entre la fuerza de los músculos del brazo y la espalda contesta al segundo objetivo establecido para esta investigación.

Estos datos coinciden con los resultados publicados por Blais y Trilles (5) y Lech et al. (7) quienes recomiendas una mayor concreción en la planificación del entrenamiento funcional para judokas de alto rendimiento, puesto que permiten una mejora en el rendimiento motor durante el combate de judo.

En otro estudio que involucró a 14 judokas finalistas en el Campeonato de Europa de 2005 se pudo ratificar que existen diferentes acciones específicas que requieren de elevados niveles de fuerza funcional (19). Esta necesidad de diferentes acciones durante la lucha para un alto rendimiento refuerza los resultados encontrado en el presente estudio.

La importancia del entrenamiento global del judoka de alto nivel se ha convertido recientemente en un tema de interés investigador, para poder incrementar el rendimiento físico, la protección de las lesiones, mejora de la capacitación técnica, y la preparación psicológica (18).

El presente estudio indica que la fuerza muscular de los brazos y espalda, así como la flexibilidad de la región lumbar puede incrementarse en los judokas de alto rendimiento tras un periodo de entrenamiento específico combinado con el entrenamiento técnico y funcional.

Esta información es importante ya que, según Deval et al. (20) los judokas se caracterizan por utilizar en mayor medida las técnicas de te-waza en las competiciones, con 
ALVES, K.J.; BORBA-PINHEIRO, C.J.; GURGEL DE ALENCAR, C.M.C.; CHULVI-MEDRANO, I.; DANTAS M.E.H. Fuerza muscular y flexibilidad de judokas masculinos de alto rendimiento que participaron en la liga española durante 2011. Mot. Hum. 13(1): 28-35; 2012.

un alto porcentaje de aplicación de seoi-nage y kataguruma porque estas técnicas requieren una gran fuerza de los brazos y también de flexibilidad de los músculos lumbares. Informaciones que son corroborados por otros estudios $(1,6,17,21)$ debido a que la planificación del entrenamiento de los judokas deben considerar una com- pleja interacción de las variables físicas para el acondicionamiento efectivo de estos atletas. Sin embargo, para futuros estudios se sugiere la inclusión de un grupo control para poder comparar los efectos del programa de entrenamiento propuesto en el presente estudio.

\section{REFERENCIAS BIBLIOGRÁFICAS}

1. Franchini E. Judô Desempenho Competitivo. São Paulo: Manole, 2001.

2. Verkoshasky YV. Entrenamiento deportivo: planificación e programación. Barcelona, Martinez Roca, 1990.

3. American College Sports medicine (ACSM). Progression Models in Resistance Training for Healthy Adults: Position Stand, 2009; 687-708.

4. O'Sullivan D, Chung C, Lee K, Kim E, Kang S, Kim T, Shin I. Measurement and comparison of Taekwondo and Yongmudo turning kick impact force for two target heights. J Sports Science Medicine 2009; 8(CSSI III):13-16.

5. Blais L. and Trilles F. The progress achieved by judokas after strength training with a judo-specific machine. J Sports Science Medicine, 2006;CSSI:132-135.

6. Sterkowicz S, Garcià JMG, Lerma, FS. The importance of judo trainers' professional activities. Archives of Budo 2007; 3: 57-61.

7. Lech G, Tyka A, Palka T, Krawczyk R. Effect of physical endurance on fighting and the level of sports performance in junior judokas. Archives of Budo 2010; 6 (1):1-6.

8. Franchini E, Miarka B, Matheus L, Del Vecchio F.B. Endurance in judogi grip strength tests: Comparison between elite and non-elite judo players. Archives of Budo 2011;7(1):1-4.

9. Drid P, Ostojic SM, Vujkov S, Purkovic S, Trivic T, Stojanovic M. Physiological adaptations of a specific muscle-imbalance reduction training programme in the elite female judokas. Archives of Budo 2011;7(2): 61-64.

10. World Medical Association. Declaration of Helsinki. Ethical Principles for Medical Research Involving Human Subjets. 59th WMA Genral Assembly, Seoul, 2008.

11. Marfell-Jones M, Olds T, Stewart A, Carter L. International standards for anthropometric assessment. ISAK: Potchefstroom, South Africa, 2006.
12. Wells KF and Dillon EK. The sit and reach: a test of back and leg flexibility. Res Q Exerc Sport 1952; 23:115-8.

13. Sánchez ÁG, Domínguez AS, Turpin JAP, Tormo JMC, Llorca CS. Importance of hand-grip strength as an indicator for predicting the results of competitions of young judokas. Archives of Budo 2011;7(3):167-172.

14. O'Donovan O, Cheung J, Catley M, McGregor AH, Strutton AH An investigation of leg and trunk strength and reaction times of hardstyle martial arts practitioners. J Sports Science Medicine 2006; CSSI: $5-12$.

15. Dantas EHM. A prática da preparação física. 5 ed - Rio de Janeiro, Shape, 2003.

16. Dantas EHM, Salomão PT, Vale RGS, Achour Júnior A, Simão R, Figueiredo NMA. Scale of perceived exertion in the flexibility (PERFLEX): a dimensionless tool to evaluate the intensity? Fit Perf J, 2008;7(5):289-94.

17. Sterkowicz S, Lech G, Chwala W, Ambrozy T, Jaworski J, Klys A. Muscle strength in young judo contestants vs. untrained subjects. Archives of Budo 2011;7( 3):179-184.

18. Sterkowicz S, Lech G, Almansba R. The course of fight and the level of sports achievements in judo. Achives of Budo 2010;6(1):1-6.

19. Boguszewski D y Boguszewska K. Dynamics of judo contests performed by finalists of European Championships (Rotterdam 2005). Archives of Budo 2006;2:40-44.

20. Deval VC, García JMG, Monteiro FL. Análisis de las acciones técnicas de los judokas cadetes participantes en el campeonato de España. Rev Int Cienc del Deporte. 2009;15(5):64-80 http://www.cafyd.com/ REVISTA/01505.pdf.

21. Carballeira E, Iglesias E. Efectos agudos del enfrentamiento en judo: análisis multiparamétrico. Motricidad. European J Human Movement, 2007;19:117-144.

\section{Dirigir Correspondencia a:}

João Alves Kimo

kimobr@hotmail.com

Calle Benicarlo, 27 pt 12cp 46020. Valencia.

España. 